Published in final edited form as:

Curr Opin Anaesthesiol. 2017 February ; 30(1): 113-117. doi:10.1097/ACO.0000000000000422.

\title{
Mortality and Cost of Acute and Chronic Kidney Disease After Cardiac Surgery
}

\author{
Nicholas Lysak, MD ${ }^{1}$, Azra Bihorac, MD MS ${ }^{2}$, and Charles Hobson, MD MHA ${ }^{3}$ \\ 1 Department of Surgery, University of Florida, Gainesville, FL \\ 2 Department of Medicine, University of Florida, Gainesville, FL \\ ${ }^{3}$ Department of Health Services Research, Management, and Policy, University of Florida, \\ Gainesville, FL
}

\begin{abstract}
Purpose-Acute and chronic kidney diseases (AKI and CKD) have far-reaching implications for surgical patients in regards to postoperative outcomes and hospital cost. We review the recent literature on the effects of AKI and CKD on morbidity, mortality, and resource utilization among cardiac surgery patients.
\end{abstract}

Recent findings-Both AKI and CKD increase the risk for short and long-term mortality, morbidity, length of stay, and hospital cost among postoperative patients, with increasing disease stage correlating with worse outcomes. Even the mildest forms of AKI (RIFLE-R) and CKD (proteinuria without an observed reduction in eGFR) demonstrate worse clinical outcomes compared to patients with no AKI or CKD. Outcomes are worse even in patients who achieve full renal recovery before hospital discharge. These complications dramatically increase ICU length of stay, hospital length of stay, resource utilization, and both in-hospital and post-discharge costs, as evidenced by lower rates of discharges to home.

Summary-Acute and chronic kidney diseases remain prevalent, morbid, and costly conditions for cardiac surgery patients. Better risk stratification, early diagnosis, and earlier interventions are needed to prevent the consequences of these diseases.

\section{Keywords}

Acute kidney injury; AKI; chronic kidney disease; CKD; cardiac surgery; mortality; cost

\section{Introduction}

Acute and chronic kidney diseases are associated with increased short and long term morbidity and mortality across multiple patient populations ${ }^{1-6}$. Both preoperative chronic

\footnotetext{
Author: Nicholas Lysak MD, Department of Surgery, 1600 SW Archer Road, Rm 6130, Gainesville, FL 32610, Telephone: (352) 265-0916, nicholas.lysak@surgery.ufl.edu. Co-author: Charles Hobson MD MHA, Department of Surgery, NF/SG VAMC,

Gainesville, FL 32608, Telephone: 352-514-2854, chobson@ufl.edu. Corresponding author: Azra Bihorac MD MS, Department of Medicine, PO Box 100254, Gainesville, FL 32610-0254, Telephone: (352) 273-9009, Fax: (352) 392-7029,

abihorac@medicine.ufl.edu.

Conflicts of Interest: No authors report conflicts of interest.
} 
kidney disease (CKD) and postoperative acute kidney injury (AKI) have been linked to worse operative outcomes, increased mortality and more resource utilization ${ }^{7,8}$. Cardiac surgery patients are particularly prone to the development of postoperative AKI given the disease burden at the time of surgery, the nature of the procedures and the common use of cardiopulmonary bypass $(\mathrm{CPB})^{9-11}$. Patients with preoperative chronic kidney disease (CKD) often have higher incidences of cardiovascular comorbidities that may require operative intervention, such as coronary artery disease, silent myocardial ischemia, valvular disease and arrhythmias 4,12 . Despite ongoing efforts to develop diagnostic and interventional strategies to identify, mitigate and even prevent postoperative renal dysfunction, both CKD and AKI remain prevalent and costly conditions among cardiac surgical patients.

\section{Outcomes - Mortality}

Acute kidney injury has repeatedly been shown to increase short and long term mortality across multiple patient populations, both surgical and non-surgical ${ }^{2,5}$, 7, 13-17. Postoperative AKI is associated with increased in-hospital mortality, with increasing severity of AKI stage correlating with worse outcomes ${ }^{1-3,17}, 18$. Machado et al demonstrated that among postoperative $\mathrm{CABG}$ patients with elevated preoperative serum creatinine, $76 \%$ of those who developed stage 3 AKI required RRT, of which $66 \%$ died within 30 days ${ }^{19}$. Even patients with the mildest forms of CSA-AKI, RIFLE-R class, have higher rates of in-hospital mortality and length of stay ${ }^{8}$. Higher long term mortality persists for postoperative AKI patients when followed as long as up to ten years, with survival differences as much as $44 \%$ versus $63 \%$ for AKI and no AKI respectively at 10 years $1,2,20$. This long term mortality differential persists even for those patients with postoperative AKI who have complete recovery of renal function by the time of discharge from the hospital ${ }^{1,16,17}$.

A significant prognostic indicator appears to be the duration of CSA-AKI. Brown et al showed that longer durations of AKI were associated with both increased in-hospital mortality (15.3\% for AKI lasting 7 days or longer compared to $4.1 \%$ for AKI lasting 1-2 days) and reduced 5-year survival (HR 3.40 for AKI lasting 7 days or longer compared to HR 1.66 for AKI lasting 1-2 days) ${ }^{21}$. Similarly, Swaminathan et al demonstrated that early renal recovery after AKI had better long term survival, with the most significant predictor of 1 -year survival being the percent decrease of serum creatinine within 24 hours of its peak ${ }^{22}$.

Patients with CKD are known to have higher rates of cardiovascular comorbidities such as coronary artery disease, arrhythmias, silent ischemia, and valve disease ${ }^{4,12}$. It has recently been shown that patients with postoperative AKI have high rates of cardiovascular specific mortality after major surgery, similar to that known to occur in patients with CKD. ${ }^{14}$. CKD has been shown to be an independent predictor of in-hospital mortality and morbidity, and worse clinical outcomes are associated with more severe stages of $\mathrm{CKD}^{6,23-25}$. In a cohort of 7621 patients undergoing CABG, valve, or combined procedures, the odds ratios of inhospital mortality were $1.45,2.8$, and 7.5 for CKD stages 2,3 , and 4 respectively. In this same cohort, the hazard ratios for late mortality were 1.2, 1.95, and 3.2 for CKD stages 2, 3, and 4 respectively when followed out to a median of 42 months ${ }^{23}$. Hedley et al demonstrated an increase in operative mortality of $35-43 \%$ for every decrease of $10 \mathrm{~mL} / \mathrm{min} / 1.73 \mathrm{~m}^{2}$ in 
preoperative estimated glomerular filtration rate (eGFR) in patients undergoing $\mathrm{CABG}^{24}$. Even minimal increases in preoperative serum creatinine or decreases in eGFR that still remain within established normal reference ranges have been associated with increased mortality, infection, RRT requirement, and cerebrovascular accidents ${ }^{26}$.

As previously described, patients with preoperative renal dysfunction are at an increased risk of developing postoperative $\mathrm{AKI}^{19,27}$, even those patients who exhibit proteinuria without an observed decrease in eGFR ${ }^{28,29}$. Given the increased mortality and morbidity associated with CKD and AKI independently, these effects of these complications are worse when they occur together $6,7,14$.

\section{Outcomes - Morbidity}

Postoperative AKI is associated with significant morbidity in addition to mortality. Patients with AKI also had higher rates of other postoperative complications, including infection, mechanical ventilation duration, and cerebrovascular and cardiovascular events $7,15,16,30$. Hansen et al calculated the 5 year risk of a composite cardiovascular endpoint of myocardial infarction, heart failure, or stroke to be $24.9 \%$ for patients with postoperative AKI after cardiac surgery compared to $12.1 \%$ for patients with no $\mathrm{AKI}^{30}$. Among a large single-center cohort, $79 \%$ of patients who developed AKI also developed at least one other postoperative complication after major surgery ${ }^{7}$.

The risk of developing CKD after episodes of AKI has been shown to be increased in patients with previously normal renal function and increases with worsening severity of AKI class $^{17,31,32}$. Coca et al demonstrated pooled hazard ratios of 8.8 and 3.1 for CKD and end stage renal disease (ESRD) respectively among all surgical patients with postoperative $\mathrm{AKI}^{31}$. In a large retrospective cohort of major surgical patients, only $56 \%$ of those who developed postoperative AKI achieved full renal recovery by the time of hospital discharge ${ }^{16}$. Not surprisingly, patients with the most severe cases of AKI are at the highest risk for long term renal dysfunction, with $80 \%$ of those requiring acute RRT developing CKD stage 3 or worse at 30 months in a single cohort study of both medical and surgical AKI patients ${ }^{33}$. Even among pediatric patients, whose healing and regenerative capabilities exceed those of adults, up to $10 \%$ of those with AKI were shown to have some degree of CKD 1-3 years after their AKI episode ${ }^{34}$.

\section{Cost}

In addition to mortality and morbidity, postoperative AKI contributes significantly to increased hospital lengths of stay, cost, and resource utilization. An early study, published before the adoption of consensus definitions of AKI, estimated that one episode of hospitalbased AKI resulted in nearly $\$ 7500$ in excess costs and that hospital-based AKI resulted in annual expenditures which exceed $\$ 10$ billion $^{35}$. In a large cohort of patients undergoing major surgery, patients who developed postoperative AKI incurred a total hospital cost 159\% higher than those patients who did not suffer renal dysfunction, with a mean adjusted cost of $\$ 42,600$ compared to $\$ 26,700$. In this study median costs were highest for patients with AKI who died, followed by patients with AKI who survived, and lowest for patients with no AKI. 
(Figure $1 \mathrm{~A}$ ). These patients had mean ICU and total hospital lengths of stay of 6 days and 12 days respectively, compared to 2 days and 5 days for those patients who did not develop AKI. The costs increased proportionally with increasing severity of AKI, with additional attributable costs of $\$ 10,700, \$ 21,400$, and $\$ 38,200$ for RIFLE classes -R, -I, and -F respectively ${ }^{7}$. In this study the adjusted cumulative incremental cost of individual postoperative complications was also examined, for patients with and without postoperative AKI. While all complications were associated with higher costs, a concurrent episode of AKI dramatically increased the cumulative incremental cost, especially for patients with pulmonary and cardiovascular complications. (Figure $1 \mathrm{~B}$ ). Similar results were shown by Dasta et al in post-CABG patients, where total postoperative cost averaged $\$ 37,674$ for patients with CSA-AKI compared to $\$ 18,463$ for those without, as well as increased ICU and total hospital lengths of stay, all of which increased proportionally with worsening AKI class $^{8}$. The increased costs were observed across various subcategories of care including the ICU, medical supply, laboratory, pharmacy, and respiratory services.

For patients undergoing transcatheter aortic valve replacement (TAVR) in Great Britain, AKI was among the postoperative complications with the highest attributable cost. For stage 3 AKI the additional $€ 20,468$ incurred was second only to life threatening bleeding from a non-access site, and was more expensive than requiring the placement of a second valve $\mathrm{e}^{36}$. Similar results were observed in a recent study of TAVR patients in the United States, with hospital lengths of stay twice as long and costs $50 \%$ higher for patients who sustained postoperative $\mathrm{AKI}^{37}$.

Patients with CKD also sustain higher costs and resource utilization in the perioperative setting. In a cohort of major vascular surgery patients the presence of either CKD or postoperative AKI contributed to a $23 \%$ higher total hospital costs, with adjusted incremental costs of $\$ 8,900$ and $\$ 9,100$ respectively. When both of these conditions were present, hospital costs were $49 \%$ higher with adjusted incremental cost of $\$ 19,100^{38}$.

The cost of acute and chronic kidney disease for surgical patients is not limited to that attributable to the hospital stay. These patients also incur higher costs after hospital discharge. In a study of cardiothoracic surgery patients, $90 \%$ of patients without postoperative AKI were discharged to their home from the hospital compared to only $68 \%$ of patients who developed AKI during their hospitalization ${ }^{1}$. Those who did not return home were discharged either to a long term acute care facility, skilled nursing facility, or some other form of short term rehabilitation center. These discharge dispositions burden patients with financial hardships that are not captured in hospital cost reports, along with the intangible costs associated with not returning home directly. While reduced lengths of stay may improve hospital costs, the costs and resource utilization that the patient experiences may not be significantly improved overall but simply shifted more towards the postdischarge setting 39 .

\section{Discussion}

Chronic kidney disease and acute kidney injury are common postoperative conditions, whose pervasiveness is now apparent with the adoption of consensus definitions of AKI, and 
whose impacts are being described in the studies that now use these consensus definitions. Postoperative complications have been shown to be more important overall than preoperative risks and intraoperative factors in determining short and long term survival after surgery ${ }^{40}$. Cardiac surgery patients in particular are more likely to have pre-existing CKD and are at higher risk for the development of postoperative AKI than other surgery patients.

Preoperative screening of patients undergoing cardiac surgery, to identify those patients who are at risk for postoperative AKI including especially those patients with preexisting CDK, is essential if the impact of these conditions is to be minimized. Appropriate perioperative and postoperative management of the cardiac surgery patient is also important. The large increases in cost and mortality that result from the concurrent occurrence of AKI with other postoperative complications emphasize the importance of identifying and treating preoperative CKD and preventing postoperative AKI if possible. Acute and chronic kidney diseases remain prevalent and morbid conditions for cardiac surgical patients. Identification of preoperative $\mathrm{CKD}$, better risk stratification for the patient about to undergo cardiac surgery, better preventive strategies for perioperative AKI, earlier identification and intervention for the patient who sustains postoperative AKI, and ultimately better therapies for those patients with either CKD or AKI are needed.

\section{Acknowledgments}

Source of Funding: AB is supported by grant R01 GM-110240 and P50 GM-111152 by National Institute of General Medical Sciences - National Institutes of Health. The content is solely the responsibility of the author and does not necessarily represent the official views of the National Institutes of Health. NL and CH report no grant funding.

\section{References}

*1. Hobson CE, et al. Acute kidney injury is associated with increased long-term mortality after cardiothoracic surgery. Circulation. 2009; 119:2444-53. The single-center retrospective review showed that cardiac surgery patients who developed postoperative AKI had longer ICU and hospital lengths of stay, and increased short and long-term mortality. Patients with complete renal recovery after AKI still had an increased adjusted hazard ratio for death of 1.28 (95\% CI 1.11 to 1.48) compared with patients without AKI. [PubMed: 19398670]

2. Lafrance JP, Miller DR. Acute kidney injury associates with increased long-term mortality. J Am Soc Nephrol. 2010; 21:345-52. [PubMed: 20019168]

3. Arnaoutakis GJ, et al. RIFLE criteria for acute kidney injury in aortic arch surgery. $\mathbf{J}$ Thorac Cardiovasc Surg. 2007; 134:1554-60. discussion 1560-1. [PubMed: 18023682]

4. Cooper WA, et al. Impact of renal dysfunction on outcomes of coronary artery bypass surgery: results from the Society of Thoracic Surgeons National Adult Cardiac Database. Circulation. 2006; 113:1063-70. [PubMed: 16490821]

5. Mathew A, et al. Chronic kidney disease and postoperative mortality: a systematic review and metaanalysis. Kidney Int. 2008; 73:1069-81. [PubMed: 18288098]

6. Litmathe J, Kurt M, Feindt P, Gams E, Boeken U. The impact of pre- and postoperative renal dysfunction on outcome of patients undergoing coronary artery bypass grafting (CABG). Thorac Cardiovasc Surg. 2009; 57:460-3. [PubMed: 20013618]

7. Hobson C, et al. Cost and Mortality Associated With Postoperative Acute Kidney Injury. Ann Surg. 2015; 261:1207-14. [PubMed: 24887982]

8. Dasta JF, Kane-Gill SL, Durtschi AJ, Pathak DS, Kellum JA. Costs and outcomes of acute kidney injury (AKI) following cardiac surgery. Nephrol Dial Transplant. 2008; 23:1970-4. [PubMed: 18178605] 
9. Seabra VF, Alobaidi S, Balk EM, Poon AH, Jaber BL. Off-pump coronary artery bypass surgery and acute kidney injury: a meta-analysis of randomized controlled trials. Clin J Am Soc Nephrol. 2010; 5:1734-44. [PubMed: 20671222]

10. Mangano CM, et al. Renal dysfunction after myocardial revascularization: risk factors, adverse outcomes, and hospital resource utilization. The Multicenter Study of Perioperative Ischemia Research Group. Ann Intern Med. 1998; 128:194-203. [PubMed: 9454527]

11. Bellomo R, et al. The pathophysiology of cardiac surgery-associated acute kidney injury (CSAAKI). Int J Artif Organs. 2008; 31:166-78. [PubMed: 18311733]

12. Lassnigg A, et al. Minimal changes of serum creatinine predict prognosis in patients after cardiothoracic surgery: a prospective cohort study. J Am Soc Nephrol. 2004; 15:1597-605. [PubMed: 15153571]

13. Gaber AO, et al. Cross-sectional and case-control analyses of the association of kidney function staging with adverse postoperative outcomes in general and vascular surgery. Ann Surg. 2013; 258:169-77. [PubMed: 23478526]

*14. Ozrazgat-Baslanti T, et al. Acute and Chronic Kidney Disease and Cardiovascular Mortality After Major Surgery. Ann Surg. 2016 Acute and chronic kidney diseases were associated with longterm cardiovascular-specific mortality after major surgery.

15. O'Connor ME, Kirwan CJ, Pearse RM, Prowle JR. Incidence and associations of acute kidney injury after major abdominal surgery. Intensive Care Med. 2016; 42:521-30. [PubMed: 26602784]

16. Bihorac A, et al. Long-term risk of mortality and acute kidney injury during hospitalization after major surgery. Ann Surg. 2009; 249:851-8. [PubMed: 19387314]

**17. Xu JR, et al. Risk Factors for Long-Term Mortality and Progressive Chronic Kidney Disease Associated With Acute Kidney Injury After Cardiac Surgery. Medicine (Baltimore). 2015; 94:e2025. The prospective study showed mortality and risk of progressive chronic kidney disease increased with worsening stage of postoperative AKI. Reduced survival was shown even among patients who achieved full renal recovery. [PubMed: 26559305]

18. $\mathrm{Hu}$ J, et al. Global Incidence and Outcomes of Adult Patients With Acute Kidney Injury After Cardiac Surgery: A Systematic Review and Meta-Analysis. J Cardiothorac Vasc Anesth. 2016; 30:82-9. [PubMed: 26482484]

19. Machado MN, Nakazone MA, Maia LN. Acute kidney injury based on KDIGO (Kidney Disease Improving Global Outcomes) criteria in patients with elevated baseline serum creatinine undergoing cardiac surgery. Rev Bras Cir Cardiovasc. 2014; 29:299-307. [PubMed: 25372901]

20. Najjar M, Salna M, George I. Acute kidney injury after aortic valve replacement: incidence, risk factors and outcomes. Expert Rev Cardiovasc Ther. 2015; 13:301-16. [PubMed: 25592763]

21. Brown JR, Kramer RS, Coca SG, Parikh CR. Duration of acute kidney injury impacts long-term survival after cardiac surgery. Ann Thorac Surg. 2010; 90:1142-8. [PubMed: 20868804]

22. Swaminathan $M$, et al. Impact of early renal recovery on survival after cardiac surgery-associated acute kidney injury. Ann Thorac Surg. 2010; 89:1098-104. [PubMed: 20338313]

23. Howell NJ, et al. Mild renal dysfunction predicts in-hospital mortality and post-discharge survival following cardiac surgery. Eur J Cardiothorac Surg. 2008; 34:390-5. discussion 395. [PubMed: 18502144]

24. Hedley AJ, et al. Impact of chronic kidney disease on patient outcome following cardiac surgery. Heart Lung Circ. 2010; 19:453-9. [PubMed: 20418160]

25. Charytan DM, Yang SS, McGurk S, Rawn J. Long and short-term outcomes following coronary artery bypass grafting in patients with and without chronic kidney disease. Nephrol Dial Transplant. 2010; 25:3654-63. [PubMed: 20551089]

26. Shavit L, et al. Influence of minimal changes in preoperative renal function on outcomes of cardiac surgery. Kidney Blood Press Res. 2012; 35:400-6. [PubMed: 22555290]

27. Hougardy JM, et al. Chronic kidney disease as major determinant of the renal risk related to onpump cardiac surgery: a single-center cohort study. Acta Chir Belg. 2016:1-8.

28. Huang TM, et al. Preoperative proteinuria predicts adverse renal outcomes after coronary artery bypass grafting. J Am Soc Nephrol. 2011; 22:156-63. [PubMed: 21115618]

**29. Li SY, Chuang CL, Yang WC, Lin SJ. Proteinuria predicts postcardiotomy acute kidney injury in patients with preserved glomerular filtration rate. J Thorac Cardiovasc Surg. 2015; 149:894-9. 
The mildest degrees of baseline renal dysfunction, characterized by proteinuria without a decline in eGFR, still carried an increased risk of postoperative AKI after cardiac surgery. [PubMed: 25433643]

30. Hansen MK, et al. Acute Kidney Injury and Long-term Risk of Cardiovascular Events After Cardiac Surgery: A Population-Based Cohort Study. J Cardiothorac Vasc Anesth. 2015; 29:61725. [PubMed: 25575408]

31. Coca SG, Singanamala S, Parikh CR. Chronic kidney disease after acute kidney injury: a systematic review and meta-analysis. Kidney Int. 2012; 81:442-8. [PubMed: 22113526]

32. Rydén L, Sartipy U, Evans M, Holzmann MJ. Acute kidney injury after coronary artery bypass grafting and long-term risk of end-stage renal disease. Circulation. 2014; 130:2005-11. [PubMed: 25239439]

33. Duran PA, Concepcion LA. Survival after acute kidney injury requiring dialysis: long-term follow up. Hemodial Int. 2014; 18(Suppl 1):S1-6.

34. Mammen C, et al. Long-term risk of CKD in children surviving episodes of acute kidney injury in the intensive care unit: a prospective cohort study. Am J Kidney Dis. 2012; 59:523-30. [PubMed: 22206744]

35. Chertow GM, Burdick E, Honour M, Bonventre JV, Bates DW. Acute kidney injury, mortality, length of stay, and costs in hospitalized patients. J Am Soc Nephrol. 2005; 16:3365-70. [PubMed: 16177006]

*36. Gutmann A, et al. Analysis of the additional costs of clinical complications in patients undergoing transcatheter aortic valve replacement in the German Health Care System. Int J Cardiol. 2015; 179:231-7. Postoperative AKI had the second highest attributable cost among complications after TAVR, behind only life threatening bleeding. It was more costly than requiring a second valve placement. [PubMed: 25464455]

37. Kong WY, Yong G, Irish A. Incidence, risk factors and prognosis of acute kidney injury after transcatheter aortic valve implantation. Nephrology (Carlton). 2012; 17:445-51. [PubMed: 22390156]

*38. Huber M, et al. Mortality and Cost of Acute and Chronic Kidney Disease after Vascular Surgery. AKI or CKD contributed an additional $23 \%$ higher hospital cost after major vascular surgery, while both conditions present simultaneously increased costs by 49\%. Ann Vasc Surg. 2016; 30:72-81. e1-2.

39. Hoste EA, De Corte W. Clinical consequences of acute kidney injury. Contrib Nephrol. 2011; 174:56-64. [PubMed: 21921609]

40. Khuri SF, et al. Determinants of long-term survival after major surgery and the adverse effect of postoperative complications. Ann Surg. 2005; 242:326-41. discussion 341-3. [PubMed: 16135919] 


\section{Key Points}

1. $\mathrm{AKI}$ and $\mathrm{CKD}$ contribute to increased postoperative morbidity and mortality that increases proportionally with increasing severity of disease.

2. The mortality risk in patients with AKI is present even in those who recover full renal function.

3. Patients with preoperative CKD are at increased risk of developing postoperative $\mathrm{AKI}$, and patients with $\mathrm{AKI}$ are at increased risk of developing CKD.

4. AKI and CKD contribute to longer lengths of stay and higher hospital costs that increase with worsening disease severity. 

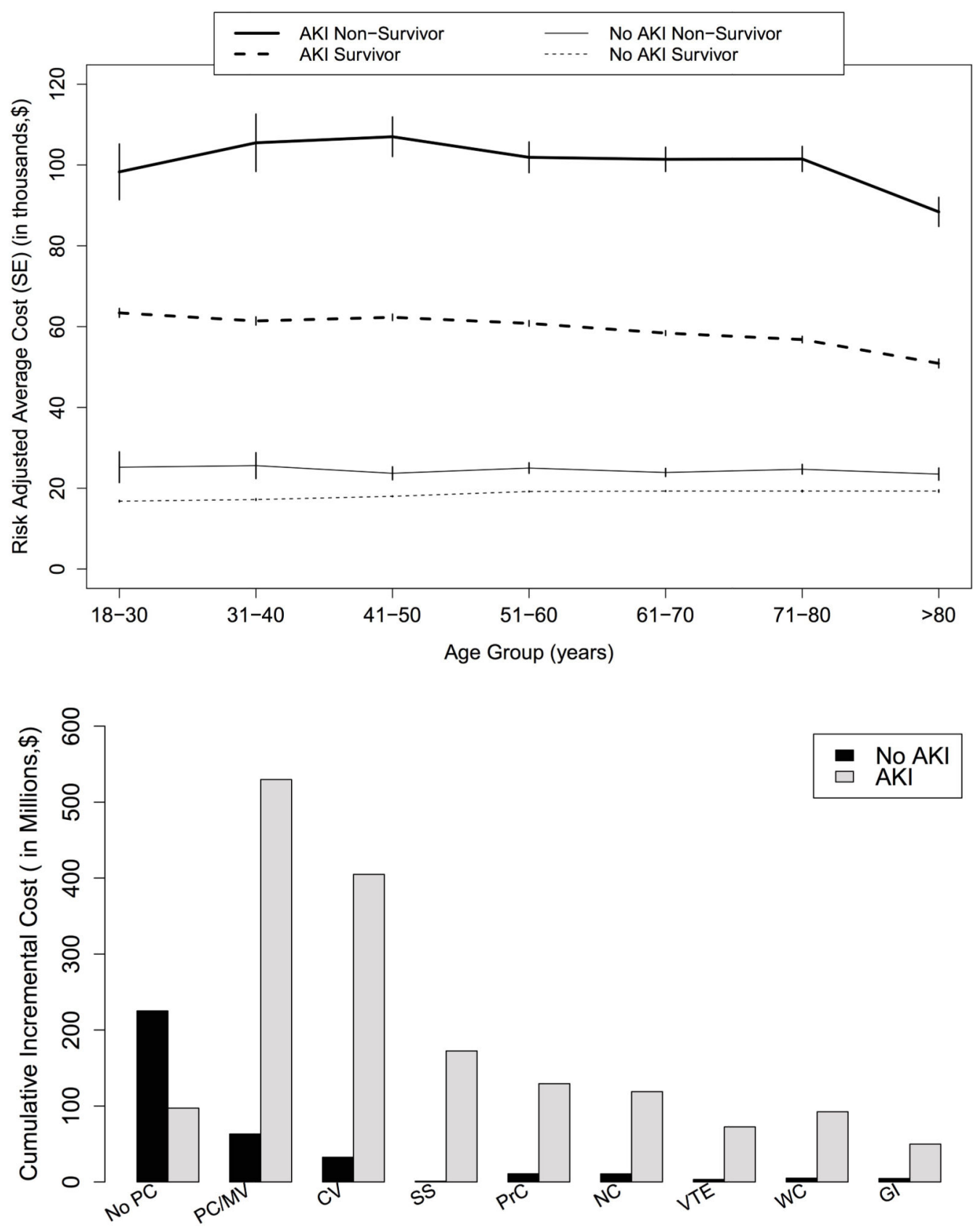

Figure 1.

A. Risk-adjusted average cost for patients stratified by 90-day survival, occurrence of acute kidney injury and age. Abbreviations: AKI, Acute kidney injury; SE, standard error. B. Risk-adjusted cumulative incremental cost of postoperative complications stratified by occurrence of acute kidney injury. Abbreviations: AKI, Acute kidney injury; No PC, No postoperative complications; PC/MV, Pulmonary complications and/or mechanical ventilation; CV, Cardiovascular complications and/or need for vasopressors; SS, Severe sepsis; NC, Neurological complications and/or delirium; VTE, Venous thromboembolism; 
WC, Mechanical wound complications and surgical infections; GI, Gastrointestinal complications. 